\title{
Blastomyces dermatitidis Antibody Responses in Serial Serum Specimens from Dogs with Blastomycosis: Comparison of Different Yeast Lysate Antigens
}

\author{
Mara Shepherd, Misty Lutes, Gene Scalarone \\ Department of Biological Sciences, Idaho State University, Pocatello, USA \\ Email: shepmara@isu.edu
}

Received 4 March 2014; revised 7 April 2014; accepted 15 April 2014

Copyright (C) 2014 by authors and Scientific Research Publishing Inc. This work is licensed under the Creative Commons Attribution International License (CC BY). http://creativecommons.org/licenses/by/4.0/

\section{Abstract}

The systemic fungal organism, Blastomyces dermatitidis causes blastomycosis in animals and humans. This study was designed to evaluate antibody detection in $\mathbf{5 5}$ serial serum specimens from 9 dogs with blastomycosis using $B$. dermatitidis yeast lysate antigens produced from two human isolates (B5896; B5931) and two dog isolates (ERC-2; T-58) with the indirect enzyme linked immunosorbent assay (ELISA; peroxidase system) to determine an optimal lysate antigen(s) for use in the ELISA to detect antibody in the dog serum specimens. The mean absorbance values when the lysate antigens were compared with respect to their ability to detect antibody in the day 0 sera from the 9 dogs were 1.024 (ERC-2), 1.351 (B5896), 1.700 (B5931) and 2.084 (T-58) respectively. All of the reagents exhibited a high level of sensitivity and in all instances the amount of antibody declined as the time interval post-treatment increased, but the T-58 lysate prepared from the dog isolate from Tennessee was the optimal reagent. We continue to evaluate antigens for $B$. dermatitidis antibody detection in different immunodiagnostic assays.

\section{Keywords}

Blastomycosis, Lysate Antigens, Antibody Detection, ELISA, Serial Dog Serum Specimens

\section{Introduction}

Blastomycosis, a systemic dimorphic fungal disease caused by Blastomyces dermatitidis, is a disease of animals and humans found mainly in North America. It is a soil saprophyte associated with sandy slightly acidic soils 
often found near a water source. The disease occurs in states that border the Ohio and Mississippi Rivers and upper Midwestern states including areas in Wisconsin and Minnesota, which are highly endemic for the disease, but is also present in other locations including certain regions of Africa and India [1]-[3]. B. dermatitidis is thermally dimorphic and hosts are infected by inhaling mycelial spores into the lung, where they convert to yeast cells and produce a primary pulmonary infection. The organism may produce an acute or chronic infection or even disseminate into other organs of the body including the skin, bones and the central nervous system. If the disease is not diagnosed or misdiagnosed and, if treatment is not instituted or delayed, it may be fatal [1] [4]-[6]. Current laboratory diagnostic methods include direct visualization, histologic, culturing the organism or immunodiagnostic methods. In certain instances these techniques may provide an unreliable diagnosis or possibly a misdiagnosis, as above [6]-[9].

Due to the increase in systemic fungal diseases researchers have begun to devote more attention to the development of immunodiagnostic assays that are reliable for blastomycosis [6] [7] [10]-[12]. For several years the thrust of research in our laboratory has also been associated with the production and comparative studies of $B$. dermatitidis yeast lysate antigens, prepared from various isolates of the fungus for the detection of antibodies in sera from immunized and infected animals [13]-[19]. Encouraging results have been obtained with novel yeast lysate antigenic preparations. As a result, studies in our laboratory have been directed to evaluate antigens from a number of different isolates of the fungus from human, animal and environmental sources in an effort to produce an efficient and reliable reagent for the immunodiagnosis of blastomycosis.

The aim of this current study was to evaluate $B$. dermatitidis yeast lysate antigens, prepared from dog and human isolates, for their ability to detect antibodies in serial serum specimens, obtained at various intervals post-treatment, from dogs with diagnosed blastomycosis. The ultimate aim of all of our comparative studies, as above, is to determine if one or more of the various $B$. dermatitidis lysate reagents can be utilized in enzyme immunoassays for the reliable diagnosis of blastomycosis.

\section{Materials and Methods}

\subsection{Lysate Antigen Preparation}

Yeast phase lysate antigens were prepared from B. dermatitidis dog isolates (ERC-2; Wisconsin and T-58; Tennessee) and human isolates (B5896 and B5931; Minnesota). The lysate reagents were prepared by a method similar to one that was previously used for the production of antigen from $H$. capsulatum [20] [22] and modified in our laboratory for $B$. dermatitidis lysate antigen production [13]. The yeast phase cells were grown for 7 days at $37 \mathrm{C}$ in a chemically defined medium in an incubator shaker, harvested by centrifugation $(700 \mathrm{x} \mathrm{g} ; 5 \mathrm{~min})$, followed by washing with distilled water, re-suspended in distilled water and then allowed to lyse for 1 day at 37 $\mathrm{C}$ in water in the incubator shaker. The preparations were then centrifuged, filter sterilized, merthiolate $(0.01 \%)$ added and stored at $4 \mathrm{C}$ for further use. Protein determinations were performed on the lysates using the BCA Protein Assay Kit (Pierce Chemical Company, Rockford, IL) and dilutions of the antigenic reagents used in the assays were based on protein concentration.

\subsection{Serum Specimens}

Fifty-five serial serum specimens from 9 dogs with diagnosed blastomycosis as well as 10 serum specimens from normal, non-infected dogs from the endemic area were provided by Dr. A.M. Legendre (University of Tennessee College of Veterinary Medicine, Knoxville, TN).

\subsection{Enzyme-Linked Immunosorbent Assay (ELISA)}

The ability of each yeast lysate reagent to detect antibodies in the above serum specimens was determined using the indirect ELISA. Each lysate antigen was diluted (2000 $\mathrm{ng}$ protein/ml) in a carbonate-bicarbonate coating buffer $(\mathrm{pH} 9.6)$ and then added to triplicate wells $(100 \mathrm{ul})$ of a NUNC 96-well microplate (Fisher-Thermo). The plates were then incubated overnight at $4 \mathrm{C}$ in a humid chamber followed by washing three times with phosphate buffered saline containing $0.15 \%$ Tween 20 (PBS-T). The serum specimens (1:2500 dilution; $100 \mathrm{ul}$ ) were added to the microplate wells and incubated for $30 \mathrm{~min}$ at $37 \mathrm{C}$ in a humid chamber. Following this incubation the wells were washed as above and $100 \mathrm{ul}$ of goat anti-dog $\operatorname{IgG}(\mathrm{H} \& \mathrm{~L})$ peroxidase conjugate (Kirkegaard and Perry, Gaithersburg, MD) was added to each well and incubated for 30 min at 37 C. 
The plates were again washed as above and $100 \mathrm{ul}$ of TMB peroxidase substrate (Pierce/Fisher-Thermo) was be added to each well and incubated for approximately $2 \mathrm{~min}$ at room temperature. The reaction was stopped by the addition of $2 \mathrm{~N}$ sulfuric acid and the absorbance read at $450 \mathrm{~nm}$ using a BIO-RAD 2550 EIA reader.

\subsection{Critical Absorbance Value}

In order to determine the detection limit of the assay, we determined the critical absorbance value (which designates positive vs. negative results) which was calculated by adding two times the standard deviation (SD) of the normal serum specimens to the mean absorbance value of the normal specimens. Two times the SD is approximately equal to the $95 \%$ confidence level.

\section{Results}

The 4 lysate antigens were compared for antibody detection in the 10 serum specimens from the normal, noninfected dogs. Absorbance values ranged from 0.242 to 0.369 (B5896; mean absorbance value: 0.284; critical absorbance value: 0.330 ), 0.221 to 0.356 (ERC-2; mean absorbance value: 0.280 ; critical absorbance value: 0.326 ), 0.246 to 0.373 (B5931; mean absorbance value: 0.303 ; critical absorbance value: 0.349 ) and 0.247 to 0.385 (T-58; mean absorbance value: 0.314 ; critical absorbance value: 0.360 ). Thus any absorbance value above the critical value is equal to antibody detection at the $95 \%$ confidence level.

Figures 1-9 represent the amount of antibody detected (mean absorbance value) by the 4 B. dermatitidis yeast lysate antigens in the dog serial serum specimens. The 9 dogs varied with regard to antibody prouctionat the various time intervals following the initiation of treatment. Thre results indicated that one antigen (T-58) exhibited the highest degree of reactivity in the comparative ELISAs with absorbance values ranging from a low of 0.336 (Dog A) to a high of 3.00 (Dogs B,E,F,H,I).

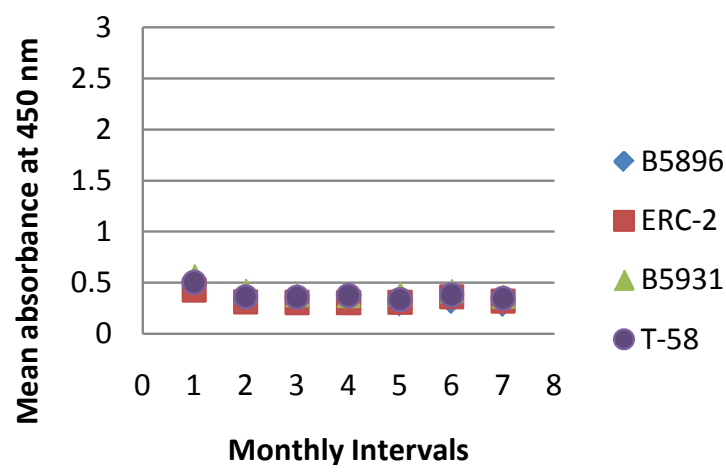

Figure 1. ELISA absorbance values from Dog A obtained with the 4 lysate antigens from 7 serial specimens at 30 day intervals following initiation of treatment.

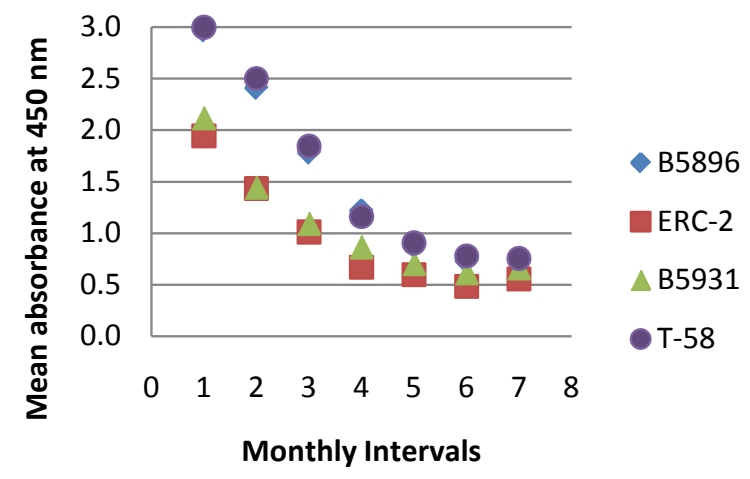

Figure 2. ELISA absorbance values from Dog B obtained with the 4 lysate antigens from 7 serial specimens at 30 day intervals following initiation of treatment. 


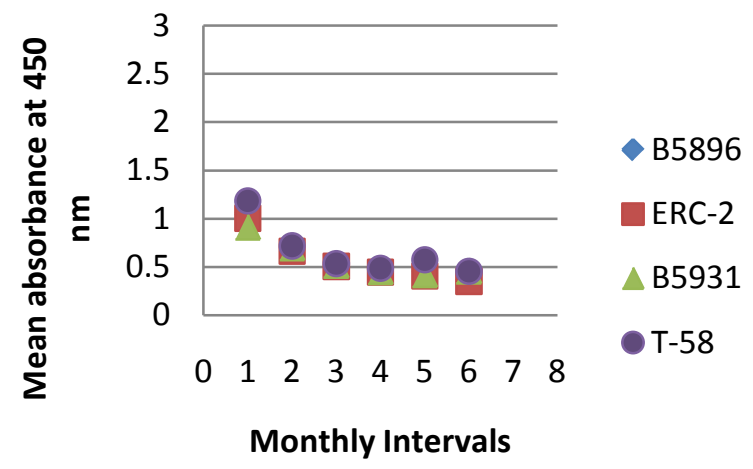

Figure 3. ELISA absorbance values from Dog C obtained with the 4 lysate antigens from 6 serial specimens at 30 day intervals following initiation of treatment.

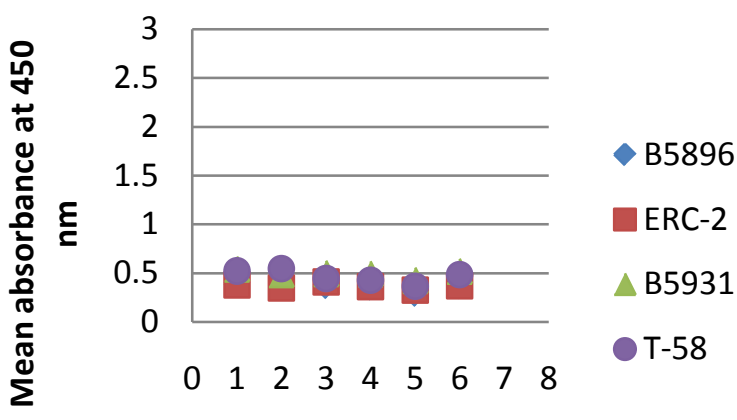

Monthly Intervals

Figure 4. ELISA absorbance values from Dog D obtained with the 4 lysate antigens from 6 serial specimens at 30day intervals following initiation of treatment.

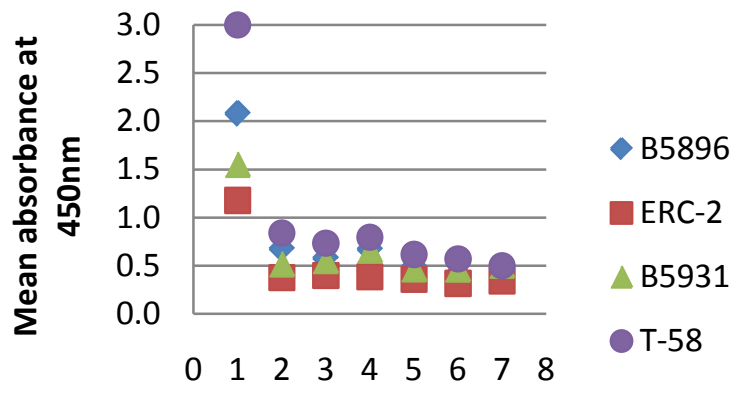

Monthly Intervals

Figure 5. ELISA absorbance values from Dog E obtained with the 4 lysate antigens from 7 serial specimens at 30 day intervals following initiation of treatment.

The highest level of antibody detection was evidenced in the day 0 specimens with mean absorbance values of 1.024 (ERC-2), 1.351 (B5896), 1.700 (B5931) and 2.084 (T-58) respectively as indicated in Figure 10. These absorbance value were well above the critical absorbance values (detection limit). There were variations in the amount of antibody present and detected in each dog, but most instances antibody declined as treatment progressed. One exception that was noted was with Dog I (Figure 9) in which the amount of antibody increased with the 4-month specimen over the 3-month specimen with absorbance values or 3.000 and 1.315 respectively. This increase was possibly due to a relapse in that dog, but we do not have treatment outcomes with any of the 


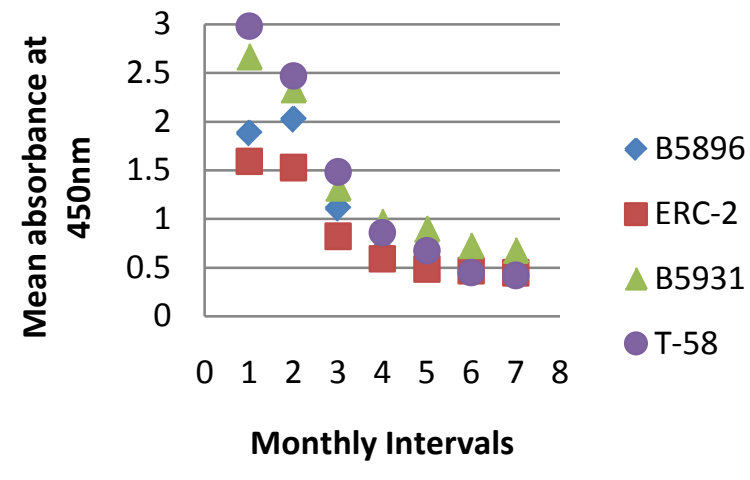

Figure 6. ELISA absorbance values from Dog F obtained with the 4 lysate antigens from 7 serial specimens at 30 day intervals following initiation of treatment.

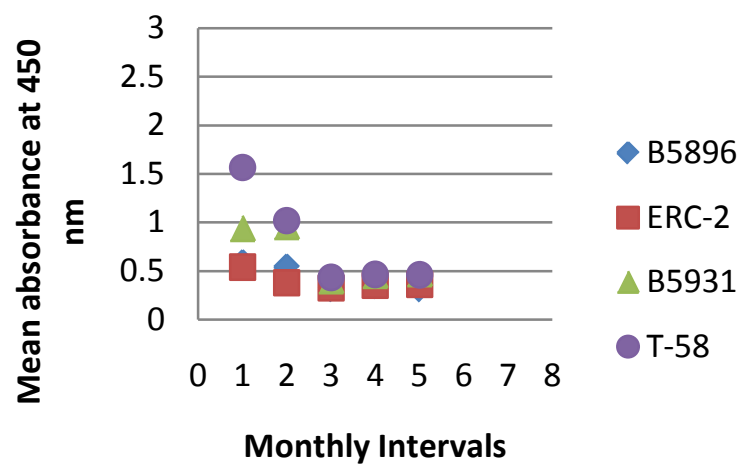

Figure 7. ELISA absorbance values from Dog G obtained with the 4 lysate antigens from 5 serial specimens at 30 day intervals following initiation of treatment.

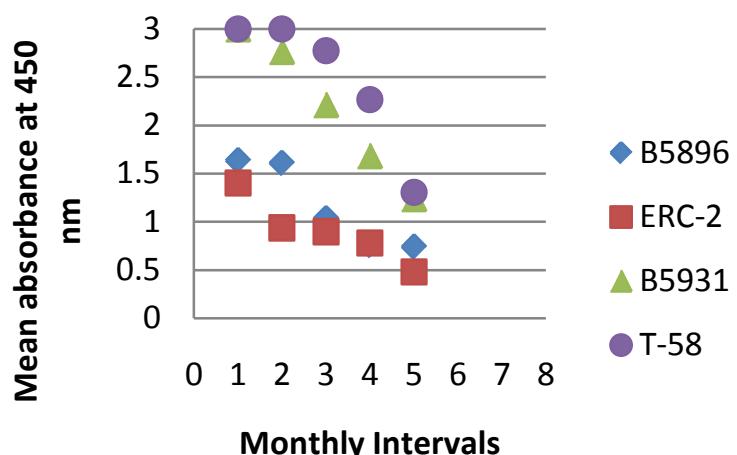

Figure 8. ELISA absorbance values from Dog $\mathrm{H}$ obtained with the 4 lysate antigens from 5 serial specimens at 30 day intervals following initiation of treatment.

dogs so we can only speculate. The amount of antibody detected in Dog D (Figure 4) was relatively constant through the treatment regimen. Was this dog immunocompromised and not able to mount an antibody response? We can only speculate on these two animals.

\section{Discussion/Conclusion}

For several years our laboratory has been interested in the production and evaluation of novel $B$. dermatitidis yeast phase lysate antigens for the detection of antibodies in serum specimens from dogs with blastomycosis. 


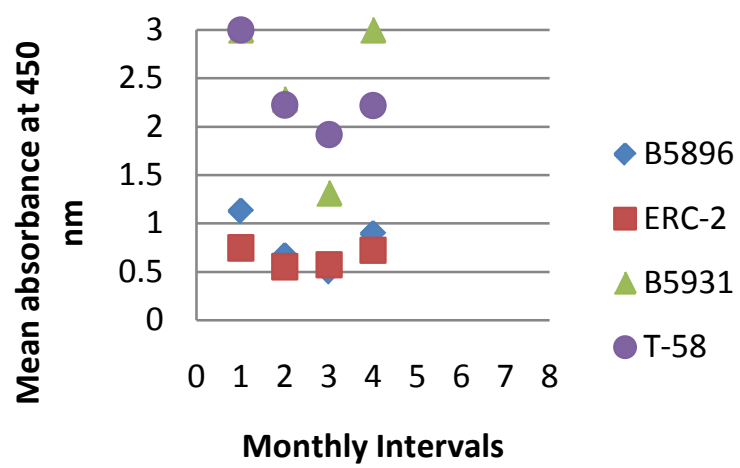

Figure 9. ELISA absorbance values from Dog I obtained with the 4 lysate antigens from 4 serial specimens at 30 day intervals following initiation of treatment.

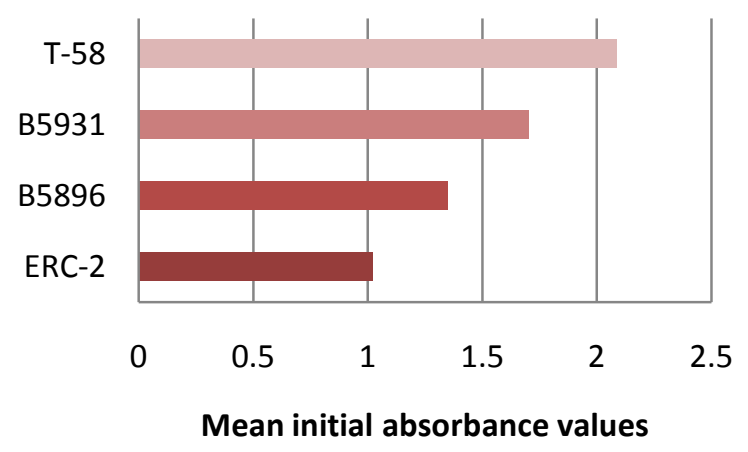

Figure 10. Mean ELISA absorbance values for antibody detection for all dogs in day 0 specimens.

We entered this area of research because of the lack of reliable methods for antibody detection in this disease. We have produced lysate antigens from a number of different isolates of the fungus and have evaluated many of the preparations for their ability to detect antibodies in dog sera. This present study was performed on serial serum specimens (pre-treatment up to 8 months post-treatment) from 9 dogs with blastomycosis in order to compare the potential for antibody detection of 4 lysate antigens produced from B. dermatitidis isolates B5896 and B5931 (human) and ERC-2 and T-58 (dog). This study allowed us to evaluate the 4 lysate reagents as the amount of antibody declined following the initiation of treatment as indicated below. Our aim is to continue to produce and evaluate our novel yeast lysates with the ultimate aim of developing an optimal immunodiagnostic antigen for the reliable diagnosis of animal and human blastomycosis.

The T-58 yeast phase lysate antigen prepared from a Tennessee dog isolate was optimal with respect to detecting antibody in the dog sera from Tennessee using the indirect ELISA (52 of the 55 sera with absorbance values greater than the critical absorbance value of 0.360 ). Previous studies using the T-58 lysate antigen to detect antibody in sera in dogs from Minnesota or Wisconsin showed similar reactivity patterns as shown above [14] [16] [17] [19]. The origin of the serum does not influence the reactivity of the lysate antigens. This is a desirable attribute in detecting antibody in blastomycosis. Therefore the T- 58 antigen appears to be promising as an immunodiagnostic antigen for the laboratory detection of blastomycosis.

\section{Acknowlegements}

This research was supported by the Department of Biological Sciences, Idaho State University.

\section{References}

[1] DiSalvo, A.F. (1998) Blastomycosis in Topley and Wilson's Microbiology and Microbial Infections. 9th Edition, Arnold Publishers, London, 337-355. 
[2] Pfaller, M.A. and Diekema, D.J. (2010) Epidemiology of Invasive Mycoses in North America. Critical Reviews in Microbiology, 36, 1-53. http://dx.doi.org/10.3109/10408410903241444

[3] Klein, B.S., et al. (1986) Isolation of Blastomyces dermatitidis in Soil Associated with a Larger Outbreak of Blastomycosis in Wisconsin. Journal of Infectious Diseases, 155, 262-268. http://dx.doi.org/10.1093/infdis/155.2.262

[4] Bradsher, R.W., Chapman, S.W. and Pappas, P.G. (2003) Blastomycosis. Infectious Disease Clinics of North America, 17, 21-40. http://dx.doi.org/10.1016/S0891-5520(02)00038-7

[5] Bariola, J.R. and Vyas, K.S. (2011) Pulmonary Blastomycosis. Seminars in Respiratory Critical Care Medicine, 32, 745-753. http://dx.doi.org/10.1055/s-0031-1295722

[6] Saccente, M. and Woods, G.L. (2010) Clinical and Laboratory Update on Blastomycosis. Clinical Microbiology Reviews, 23, 367-381. http://dx.doi.org/10.1128/CMR.00056-09

[7] McKinnell, J.A. and Pappas, P.G. (2009) Blastomycosis. New Insights into Diagnosis, Prevention, and Treatment. Clinical Chest Medicine, 30, 227-239. http://dx.doi.org/10.1016/j.ccm.2009.02.003

[8] Smith, J.A. and Kauffman, C.A. (2012) Pulmonary Fungal Infections. Respirology, 17, 913-926. http://dx.doi.org/10.1111/j.1440-1843.2012.02150.x

[9] Smith, J.A. and Kauffman, C.A. (2010) Blastomycosis. Proceedings of the American Thoracic Society, 7, $173-180$. http://dx.doi.org/10.1513/pats.200906-040AL

[10] Spector, D., et al. (2008) Antigen and Antibody Testing for the Diagnosis of Blastomycosis in Dogs. Journal of Veterinary Internal Medicine, 22, 839-843. http://dx.doi.org/10.1111/j.1939-1676.2008.0107.x

[11] B.S. Klein, B.S., et al. (2000) Canine Antibody Response to Blastomyces dermatitidis WI-1 Antigen. American Review of Veterinary Research, 61, 554-558. http://dx.doi.org/10.2460/ajvr.2000.61.554

[12] Vyas, K.S., Bariola, J.R. and Bradsher, R.W. (2008) Advances in the Serodiagnosis of Blastomycosis. Current Fungal Infection Reports, 2, 227-231. http://dx.doi.org/10.1007/s12281-008-0033-Z

[13] Johnson, S.M. and Scalarone, G.M. (1989) Preparation and ELISA Evaluation of Blastomyces dermatitidis Yeast Phase Lysate Antigens. Diagostic Microbiology and Infectious Diseases, 11, 81-86. http://dx.doi.org/10.1016/0732-8893(88)90076-4

[14] Sestero, C.M. and Scalarone, G.M. (2006) Detection of IgG and IgM in Sera from Canines with Blastomycosis Using Eight Blastomyces dermatitidis Yeast Phase Lysate Antigens. Mycopathologia, 162, 33-37. http://dx.doi.org/10.1007/s11046-006-0028-7

[15] Hatch, W.O. and Scalarone, G.M. (2009) Comparison of Colorimetric and Chemiluminescent ELISAs for the Detection of Antibodies to Blastomyces dermatitidis. Journal of Medical and Biological Sciences, 3, 1-6.

[16] VanDyke, J.L., et al. (2013) Detection of Antibodies in Serum Specimens from Dogs with Blastomycosis with Lysate Antigens Prepared from Four Blastomyces dermatitidis Dog Isolates. Individual Antigens VS Antigen Combinations. Open Journal of Veterinary Medicine, 3, 237-241. http://dx.doi.org/10.4236/ojvm.2013.34037

[17] Allison, T.R., Wright, J.C. and Scalarone, G.M. (2013) Blastomyces dermatitidis. Stability Studies on Different Yeast Lysate Antigens. Open Journal of Immunology, 3, 98-102. http://dx.doi.org/10.4236/oji.2013.33014

[18] Wright, J.C., Harrild, T.E. and Scalarone, G.M. (2012) The Use of Isoelectric Focusing Fractions of Blastomyces dermatitidis for Antibody Detection in Serum Specimens from Rabbits Immunized with Yeast Lysate Antigens. Open Journal of Veterinary Medicine, 2, 237-241. http://dx.doi.org/10.4236/ojvm.2012.24038

[19] Boyd, A.R., VanDyke, J.L. and Scalarone, G.M. (2013) Blastomyces dermatitidis Yeast Lysate Antigen Combinations: Antibody Detection in Dogs with Blastomycosis. Veterinary Medicine International, 4, Article ID: 940126.

[20] Levine, H.B., Scalarone, G.M. and Chaparas, S.D. (1977) Preparation of Fungal Antigens and Vaccines: Studies on Coccidioides Immitis and Histoplasma Capsulatum. Contributions to Microbiology and Immunology, 3, 106-125.

[21] Levine, H.B., et al. (1979) Histoplasmin-CYL, A Yeast Phase Reagent in Skin Test Studies in Humans. American Review of Respiratory Diseases, 119, 629-636.

[22] Scalarone, G.M., Levine, H.B. and Chaparas, S.D. (1978) Delayed Hypersensitivity Responses of Experimental Animals to Histoplasmin from the Yeast and Mycelial Phases of Histoplasma Capsulatum. Infection and Immunity, 21, 705-713. 